\title{
Aneurysmal Bone Cyst of Rib: A Report of a Rare Case
}

\author{
Mustafa Çalık', Saniye Göknil Çalık², Murat Serhat Aygün³, Hıdır Esme¹ \\ 'Department of Thoracic Surgery, Konya Training and Research Hospital, Konya, Turkey \\ ${ }^{2}$ Department of Emergency Medicine, Konya Training and Research Hospital, Konya, Turkey \\ ${ }^{3}$ Department of Radiology, Konya Training and Research Hospital, Konya, Turkey
}

\begin{abstract}
Introduction: Aneurysmal bone cyst $(A B C)$ is a non-neoplastic bone tumor of the skeletal system that occurs predominantly in children and young adults, accounting for $1-2 \%$ of all primary bone tumors. Any bone can be affected, but it uncommonly originates from the ribs. We present the case of a young girl with $A B C$ arising from the $6^{\text {th }}$ rib.

Case Report: A 14-year-old girl was admitted to the Emergency Department with a 10-day history of gradually worsening leftsided back pain and swelling. The pain was constant and exacerbated by movement of rotating left upper hemithorax. She had no history of trauma or prior chest surgery, and her past medical history was unremarkable. A CT of the chest revealed lytic bone lesion at the $6^{\text {th }}$ left rib. She underwent partial resection of the $6^{\text {th }}$ rib with posterolateral incision without any need for reconstruction of the chest wall.

Conclusion: Preoperative diagnosis of $A B C$ could be difficult due to its rarity and the similarity with other lesions. Here, the essence of diagnosis is to exclude other causes, but the definitive diagnosis and treatment can be performed after en bloc resection. Although a rare cause of rib tumor, $A B C$ should be kept in mind.
\end{abstract}

Keywords: Aneurysmal bone cyst, childhood, rib

Received: 12.12.2015 Accepted: 25.01.2016 Available Online Date: 16.02.2016

\section{Introduction}

Aneurysmal bone cyst (ABC) was first reported by Jaffe and Lichtenstein in 1942. ABC is a non-neoplastic bone tumor of the skeletal system that occurs predominantly in children and young adults, accounting for $1-2 \%$ of all primary bone tumors and is generally found in the metaphysis of long bones, spine, and pelvis. Any bone can be affected, but ribs are uncommonly involved. We present the case of a young girl with $A B C$ arising from the $6^{\text {th }}$ rib. (1)

\section{Case Report}

A 14-year-old girl was admitted to the Emergency Department with a 10-day history of gradually worsening left-sided back pain and swelling. The pain was constant and exacerbated by movement of rotating left upper hemithorax. She had no history of trauma or prior chest surgery, and her past medical history was unremarkable. A physical examination revealed a palpable tender mass on 6th rib at the left side of the vertebral column. The respiratory sounds were slightly decreased on the left hemithorax. Her routine laboratory test results, including complete blood count, erythrocyte sedimentation rate, tumor markers, lactate dehydrogenase, serum electrolytes, kidney function tests, and liver function tests were all within normal ranges. After initial assessment, a chest radiograph was performed, and it showed destruction of the $6^{\text {th }}$ left rib. ACT of the chest revealed a $59 \times 57-\mathrm{mm}$ multilobulated cystic bone lesion with compressive atelectatic changes in the adjacent lung parenchyma, which originated from the $6^{\text {th }}$ left rib. The mass was protruding into the hemithorax and the adjacent soft tissue. The presumptive diagnosis was neurogenic tumor. There were no other disruptive changes in other bony structures. She underwent surgery after all diagnostic investigations were completed. 


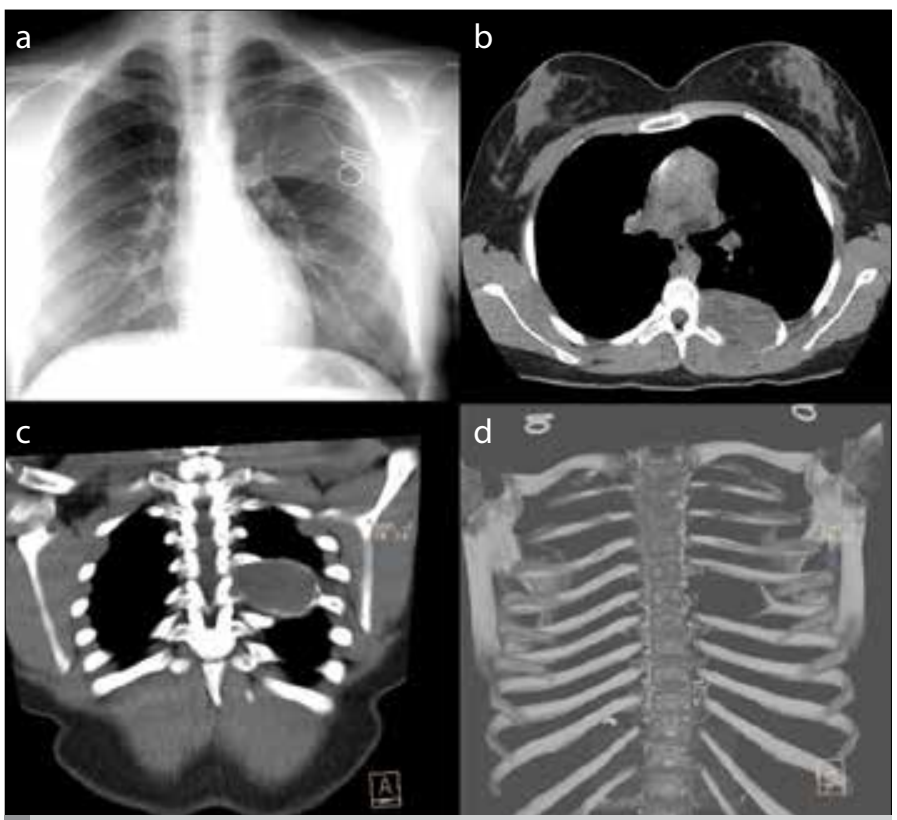

FIGURE 1. a-d. a: Chest X-ray showed destruction of posterior part of the left $6^{\text {th }}$ rib. b-c: Coronal and axial plane CT showed expansile soft tissue mass, with destruction of the cortex of the bone. $d$ : 3D-reconstructed CT image revealed expansion of the left $6^{\text {th }}$ rib.

A left-sided thoracotomy was performed. The $6^{\text {th }}$ rib was explored through an incision. The mass adhered lightly to the lung. The visceral pleura was intact. The mass was resected totally en bloc along with the affected part of the $6^{\text {th }}$ rib, underlying parietal pleura, and adjacent intercostal muscles. The chest wall was closed primarily without any grafting. $A B C$ was diagnosed pathologically with tumor-free resection margins. The patient's recovery was uneventful. She was discharged on 6th postoperative day without any complaints and was followed up for 6 months without tumor recurrence.

\section{Discussion}

ABC is not a true neoplasm, cyst, or aneurysm. The term "cyst" or "aneurysm" arises from its "blow out" radiographic appearance and blood-filled contents of the cystic spaces. Histopathologically, ABC is a destructive expansile bone lesion characterized by a reactive proliferation of connective tissue containing multiple blood-filled cavities (2). Even though the etiology and pathogenesis of $A B C$ remains unclear and controversial; trauma or circulatory disturbance due to arteriovenous malformation is greatly accepted (3). If any previous bone lesion has not been identified, it is called as "primary ABC" like in our patient. $A B C$ may be secondary to other pre-existing bone diseases, including fibrous dysplasia, giant cell tumor, and non-ossifying fibroma; and it is classified as "secondary ABC." Approximately one-third of all cases are the secondary type (4).

However, $A B C$ can be observed in the range of newborn to 71 years; approximately four-fifths of the cases are younger than 20 years, and the median age at diagnosis is 10.2 years $(1,5)$. There is no dominance of any race or gender. Long bones, spine, and pelvis are the most frequent bone sites can be affected by ABC. The ribs are an ex- tremely rare location for $\mathrm{ABC}$, only involving $2.7 \%$ of all cases. When it does, posterior or lateral parts of the ribs are primarily involved, as observed in our patient. $A B C$ can be encountered in every rib excluding the last three ribs $(1,4)$. The size of the majority of cases that have been reported in the literature is less than $10 \mathrm{~cm}$ (2).

The most common symptoms are chest pain (46\%), swelling of the chest wall (21\%), dyspnea (7\%), paraplegia (7\%), and pathologic fractures (2\%). In accordance with the literature, the main complaint was chest pain and swelling in our patient. There are no complaints in one third of cases; it is diagnosed incidentally on imaging studies such as chest X-ray or CT (4). Our patient's chest X-ray revealed destruction on the 6th left rib, and thorax CT revealed a $59 \times 57-\mathrm{mm}$ multilobulated cystic bone lesion at the 6th left rib. In particular, dyspnea frequently encountered in infants and paraplegia/pathologic fractures caused by vertebral location are rarely seen in patients with $\mathrm{ABC}(3)$.

\section{Conclusion}

During the diagnosis period, pathological fracture is common in patients with $A B C$. Presentation of only pathological fracture is not rare in patients with $\mathrm{ABC}$. Although there is no characteristic appearance, imaging techniques, including $\mathrm{CT}$ and magnetic resonance imaging, are important in differential and definitive diagnosis of rib lesions. However, ABC cannot clearly be ruled out based only on clini$\mathrm{cal}$ and radiological findings. Preoperative diagnosis of $A B C$ could be difficult due to its rarity and the similarity with other lesions. Here, the essence of diagnosis is to exclude other causes, but the definitive diagnosis and treatment can be performed after en bloc resection. Although a rare cause of rib tumor, $\mathrm{ABC}$ should be kept in mind.

Informed Consent: Written informed consent was obtained from patient who participated in this case.

Peer-review: Externally peer-reviewed.

Author Contributions: Concept - M.Ç., S.G.Ç.; Design - M.Ç., S.G.Ç.; Supervision - M.Ç., S.G.Ç., H.E.; Materials - M.Ç., S.G.Ç.; Data Collection and/or Processing - M.Ç., S.G.Ç., M.S.A.; Analysis and/or Interpretation - M.Ç., S.G.Ç.; Literature Review - M.Ç., S.G.Ç.; Writer - M.Ç., S.G.Ç.; Critical Review - M.Ç., S.G.Ç.

Conflict of Interest: The authors declared no conflict of interest.

Financial Disclosure: The authors declared that this study has received no financial support.

\section{References}

1. Güneş D, Mutafoğlu-Uysal K, Sarialioğlu F, Cakmakçi H, Olgun N. Aneurysmal bone cyst of rib presenting as a huge chest wall mass. Turk J Pediatr 2009; 51: 82-5.

2. Guo J, Liang C. A giant aneurysmal bone cyst of the rib: Case report. Oncol Lett. 2014; 7: 267-9.

3. Yasaroglu M, Ketenci B, Demirbag H, Yildirim M, Dogusoy I. Aneurysmal bone cyst of the rib: a case report. J Med Case Rep 2009; 3: 8457. [CrossRef]

4. Soyer T, Karnak I, Talim B, Tanyel FC. Aneurysmal bone cyst of the rib in a child: report of a case. Surg Today 2005; 35: 886-9. [CrossRef]

5. Yoshida K. Aneurysmal bone cyst of the rib: report of a case. Surg Today 2005; 35: 1073-5. [CrossRef] 